\title{
Sigmoid Colonic Perforation with Faecal Peritonitis due to Faecaloma
}

\author{
Ibrahim Khali1 ${ }^{1}$, Deb Proshad Paul ${ }^{2}$, Kazi Shohel Iqbal ${ }^{3}$, Debashish Das ${ }^{4}$ \\ Received: January 2, 2014 Accepted: July 5, 2014
}

\begin{abstract}
Colon perforation is an uncommon event usually caused by malignancy, diverticular disease, amoebic colitis, steroid therapy, trauma and ulcerative colitis, but stercoral perforation is very rare. Severe chronic constipation is considered to be the main causative factor in development of stercoral perforation of colon. Sometimes it can also produce catastrophic complications like colonic obstruction, faecal peritonitis and septicaemia. We report a rare case of sigmoid colonic perforation with faecal peritonitis and pneumoperitonium due to faecaloma which was diagnosed after exploratory laparotomy.
\end{abstract}

Key words: Sigmoid colonic perforation; Faecaloma; Pneumoperitoneum; Laparotomy

J Enam Med Col 2014; 4(3): 188-190

\section{Introduction}

Sigmoid colon perforation with faecal peritonitis may occur due to various causes, most commonly diverticular disease and colonic carcinoma. Stercoral peritonitis is associated with a high mortality $(35 \%)$ and morbidity rate; therefore an early diagnosis is important. ${ }^{1}$ Stercoral colitis is an inflammatory colitis related to faecal impaction which results in ischaemic pressure necrosis of the rectal and colonic wall, usually the sigmoid colon, due to increased intraluminal pressure; this leads to stercoral ulcer formation and subsequent colonic perforation. ${ }^{2}$ But other causes should be excluded. Diagnosis of an intestinal perforation can be difficult and requires high degree of suspicion and awareness. So a correct preoperative diagnosis can facilitate early surgical intervention and thus prevent or mitigate serious complications.

\section{Case report}

A 58-year-old man presented with a history of sudden severe generalised abdominal pain for two days in March, 2012 in Enam Medical College Hospital. Pain was continuous and colicky in nature and was aggravated by movement. Pain was also associated with nausea and vomiting. Patient subsequently developed abdominal distension. He had a history of chronic constipation for 7 days but no history of alteration of bowel or bladder habit or per rectal bleeding. He did not give any history of cough, haemoptysis, haematemesis, melaena, chest pain or bone pain.

On clinical examination, his pulse rate was 104 beats/min, temperature $100^{\circ} \mathrm{F}, \mathrm{BP} 100 / 60 \mathrm{~mm}$ of $\mathrm{Hg}$ and respiratory rate was normal. Signs of dehydration were present. Abdominal examination revealed restricted abdominal movement, board like rigidity, obliteration of liver dullness and absent bowel sound. Faecal impaction was found on digital rectal examination. Laboratory investigations including complete blood count, urine routine examination, serum creatinine, serum electrolytes, random blood sugar and ECG were within normal limit; but plain radiography of abdomen showed free gas shadow under both domes of diaphragm. A provisional diagnosis of duodenal ulcer perforation was made and consent was taken for

1. Assistant Professor, Department of Surgery, Enam Medical College \& Hospital, Savar, Dhaka

2. Associate Professor, Department of Surgery, Enam Medical College \& Hospital, Savar, Dhaka

3. Associate Professor, Department of Surgery, Enam Medical College \& Hospital, Savar, Dhaka

4. Associate Professor, Department of Surgery, Enam Medical College \& Hospital, Savar, Dhaka

Correspondence Ibrahim Khalil, Email: ibrahimk8@gmail.com 
laparotomy followed by repair of perforation and thorough peritoneal toileting.

On laparotomy, there was no evidence of duodenal ulcer perforation. A large oval shaped perforation was found in middle of sigmoid colon with faecal contamination. Other portions of both small and large intestines were normal except distension of caecum, ascending and transverse colon. Hartman procedure was done and specimen was sent for histopathological examination. Postoperative recovery was uneventful.

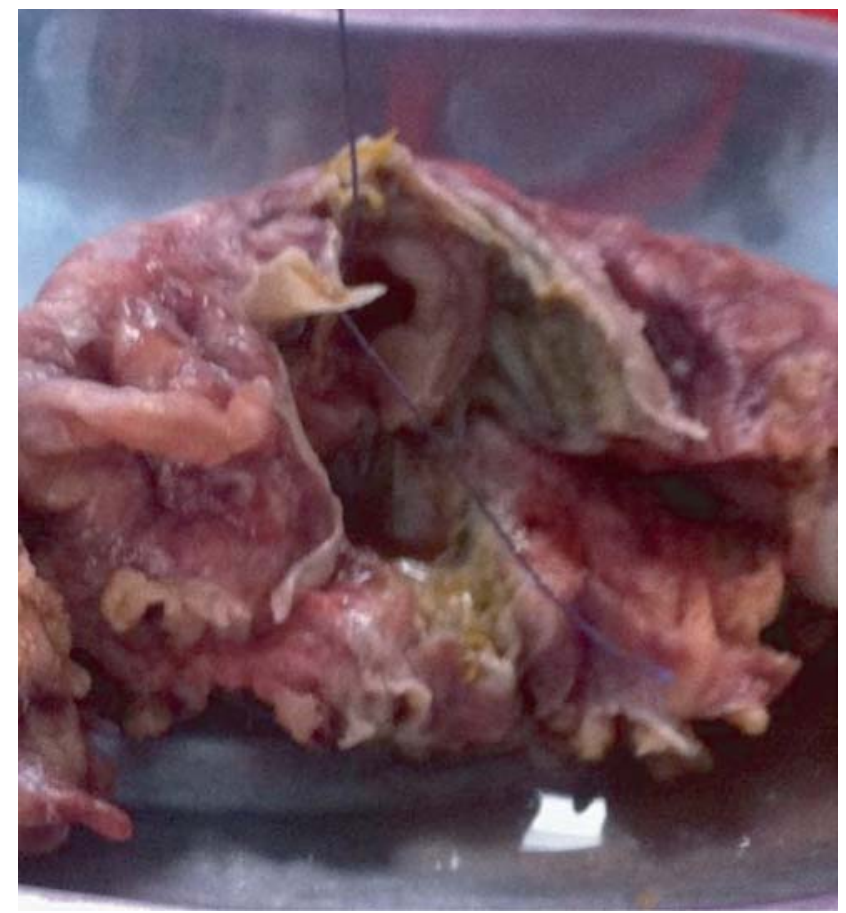

Fig1. Findings of sigmoid colonic perforation (arrow shows perforation site)

\section{Discussion}

Sigmoid colonic perforation is a rare surgical condition with fewer than 90 cases reported in the literature up to 2002. 3,4 This condition was first described by Berry in $1984^{4}$ and was defined as a colonic perforation due to pressure effect of the hard faecalomas on the wall of an otherwise normal colon, in absence of any other established pathology. ${ }^{5}$

The mean age of presentation of this disease is 59 years with age range $22-85$ years. ${ }^{6}$ Prior history of constipation is present in $60 \%$ cases of faecal impaction. ${ }^{6}$ Chronic intermittent constipation and use of nonsteroidal anti-inflammatory drugs (NSAIDS) and drugs like amitryptilline, antacid, heroine, codeine, steroids are important risk factors for sigmoid ulcer perforation. ${ }^{3,7,8}$ In this case, the patient had history of chronic constipation.

On CT scan faecal impaction is diagnosed clearly with the visualisation of the dilated rectum and colon containing dense lamellated faecal masses (faecalomas) with thin colonic walls. Findings of sigmoid colitis include colonic or rectal wall thickening involving the dilated sigmoid colon and rectum, which demonstrate faecalomas.

There is poor hydration of the faeces resulting in hard and impacted faeces, also called faecaloma. Thus intraluminal pressure is increased which causes ischaemic necrosis of the colonic wall, sigmoid colon ulcer formation and subsequently colon perforation. ${ }^{2}$ Ulceration and perforation most commonly (>90\%) occur in anterior rectum (just proximal to peritoneal reflection), the antimesenteric border of the rectosigmoid junction and the sigmoid colon ${ }^{2,3}$ due to the decreased perfusion at the antimesenteric border of sigmoid and recto-sigmoid colon ${ }^{4,2}$ and the narrow diameter of the distal colon for which it is difficult for hard stools to pass through sigmoid colon leading to increased intraluminal pressure and ischaemia. ${ }^{4}$ In this case, patient had an oval shaped perforation in the middle of the sigmoid colon with faecal contamination.

Pericolonic/perirectal fat stranding is usually seen due to colonic ischaemia or wall oedema. ${ }^{2}$ Presence of intramural or extraluminal air loculi are suggestive of colonic perforation. According to Maurer et $\mathrm{al}^{6} \mathrm{few}$ diagnostic criteria for sigmoid colon perforation are (1) a rounded or ovoid colonic perforation which is $>1 \mathrm{~cm}$ in diameter and antimesenteric in location, (2) the presence of faecalomas within the colon, protruding through perforation site or found free in the abdominal cavity and (3) pressure necrosis, ulcer formation and chronic inflammatory reaction around the perforation site microscopically.

According to these criteria, presence of any colonic pathology, such as diverticulitis, infectious process, inflammatory bowel disease or obstruction exclude the diagnosis of primary sigmoid colon perforation. Preoperative diagnosis of sigmoid colitis is important in elderly patient with severe colonic constipation or long history of using constipating drug and less commonly in 
young patients who are neurologically impaired. ${ }^{2,8} \mathrm{CT}$ scan is particularly important in preoperative diagnosis in the elderly age group since the laboratory findings may not always correlate with the actual pathology. Without prompt treatment, this condition can be fatal with $35 \%$ mortality rate. ${ }^{2}$

Stercoral peritonitis is an extreme condition followed by high morbidity and mortality rate. Clinicians should consider the possibility of sigmoid colon perforation in the patients with a history of severe or chronic constipation presenting with acute symptoms. Prompt recognition and well intensive care can increase the rate of survival significantly.

\section{References}

1. Hoch J. Stercoral peritonitis. Klinická Onkologie Zvláštní Éíslo 1999: 30-33.

2. Heffernan C, Pachter HL, Megibow AJ, Macari M. Stercoral colitis leading to fatal peritonitis: CT findings. Am J Roentgenol 2005; 184: 1189-1193.
3. Patel VG, Kalakuntla V, Fortson JK, Weaver WL, Joel MD, Hammami A. Stercoral perforation of the sigmoid colon: report of a rare case and its possible association with nonsteroidal anti-inflammatory drugs. Am Surg 2002; 68: 62-64.

4. Huang WS, Wang CS, Hsieh CC, Lin PY, Chin CC, Wang JY. Management of patients with stercoral perforation of the sigmoid colon: report of five cases. World J Gastroenterol 2006; 12: 500-503.

5. Arvind N, Gowrisankar A, Rajkumar JS. Primary stercoral perforation of the colon-rare, but deadly. Indian J Surg 2006; 68: 56.

6. Maurer CA, Renzulli P, Mazzucchelli L, Egger B, Seiler CA, Büchler MW. Use of accurate diagnostic criteria may increase incidence of stercoral perforation of the colon. Dis Colon Rectum 2000; 43: 991-998.

7. Tessier DJ, Harris E, Collins J, Johnson DJ. Stercoral perforation of the colon in a heroin addict. Int $\mathrm{J}$ Colorectal Dis 2002; 17: 435-437.

8. Hollingworth J, Alexander-Williams J. Non-steroidal antiinflammatory drugs and stercoral perforation of the colon. Ann R Coll Surg Engl 1991; 73: 337-340. 